\title{
Ethel GROFFIER, Le stratège des Lumières. Le comte de Guibert (1743-1790)
}

\section{Annie Crépin}

\section{OpenEdition \\ Journals}

Édition électronique

URL : https://journals.openedition.org/ahrf/10292

DOI : 10.4000/ahrf.10292

ISSN : $1952-403 X$

Éditeur :

Armand Colin, Société des études robespierristes

Édition imprimée

Date de publication : 15 mars 2006

Pagination : 227-229

ISSN : 0003-4436

\section{Référence électronique}

Annie Crépin, «Ethel GroffIER, Le stratège des Lumières. Le comte de Guibert (1743-1790) », Annales

historiques de la Révolution française [En ligne], 343 I janvier-mars 2006, mis en ligne le 17 novembre 2008, consulté le 23 avril 2022. URL : http://journals.openedition.org/ahrf/10292 ; DOI : https:// doi.org/10.4000/ahrf.10292

Ce document a été généré automatiquement le 23 avril 2022.

Tous droits réservés 


\section{Ethel GROFFIER, Le stratège des Lumières. Le comte de Guibert (1743-1790)}

Annie Crépin

\section{RÉFÉRENCE}

Ethel Groffier, Le stratège des Lumières. Le comte de Guibert (1743-1790), Paris, Honoré Champion, 2005, 409 p., ISBN, 2-7453-1116-6, $70 €$.

1 Les spécialistes de l'histoire militaire connaissent bien Guibert, militaire philosophe, dont la pensée novatrice et à bien des égards prémonitoire a été redécouverte à la faveur du développement de la nouvelle histoire militaire. Mais pour bien d'autres, il n'est que l'amant ingrat et égocentrique de Mademoiselle de Lespinasse, ou plutôt, il n'apparaît comme tel que parce que les Lettres de celle-ci ont connu une fortune littéraire que les ouvrages de Guibert n'ont pas eue. L'image qui apparait dans ces lettres n'est guère flatteuse pour le destinataire sans qu'on se soit beaucoup interrogé sur la subjectivité de leur auteur.

2 Forte de ce constat, Ethel Groffier, qui a enseigné le droit à l'Université de Montréal, a entrepris une réhabilitation de Guibert. Qu'on n'attende pas une thèse ou un ouvrage spécialisé sur la pensée militaire de celui-ci. L'œuvre d'E. Groffier est une synthèse clairement écrite qui a pour but de rendre justice à un homme de talent, sinon de génie, en le replaçant dans la société de son temps et en retraçant la genèse de sa pensée, d'« une aventure intellectuelle», selon les propres mots de l'auteur. C'est d'ailleurs le grand mérite de celle-ci que d'avoir analysé tous les ouvrages de Guibert même ceux qui sont mineurs et qui ne sont pas consacrés à la pensée militaire.

Le livre est divisé en trois parties. La première intitulée "Les idées-forces ", la plus longue puisqu'elle occupe la moitié de l'ouvrage, est consacrée au jeune Guibert, à sa formation intellectuelle, sociale et professionnelle et à un tableau vivement mené du 
laboratoire intellectuel dans lequel il va très vite évoluer, c'est-à-dire le monde des salons. On retrouve des notations qui, pour être connues des historiens militaires, n'en sont pas moins déterminantes : l'appartenance à un milieu de récente noblesse et aussi de petite noblesse militaire, décidément terreau d'une interrogation sur le rôle de l'armée et ses rapports avec la société. Avant même d'être introduit dans les salons, Guibert manifeste une capacité de travail extraordinaire et une originalité de pensée plus grande encore, qu'il manifeste dans son Essai général de tactique auquel E. Groffier consacre une longue analyse. Mais elle montre aussi que Guibert exprime autrement, si ce n'est avec un égal talent, ses conceptions militaro-politiques, dans une tragédie (Le Connétable de Bourbon) où surgit un patriotisme qui va déjà plus au pays qu'au monarque

4 Son essai lui ouvre les portes des salons dont celui de Julie de Lespinasse. L'auteur fait remarquer très justement que la fréquentation des salons n'a rien de frivole ni même de spécifiquement mondain mais est pour un jeune écrivain le passage obligé vers le succès et la diffusion de ses idées. D'autant que Guibert n'est pas homme de salon au sens péjoratif qu'a pris ce terme. Par réaction contre l'image traditionnelle de Guibert, E. Groffier fait de Julie de Lespinasse un portrait qui n'a rien de flatteur; maîtresse possessive, jalouse et quelque peu névrosée, « s'accrochant » à Guibert, homme solide psychologiquement parlant, peut-être plus doué pour l'amitié que pour l'amour, et dont la passion la plus intense va au service de la patrie, ce qu'il appelle «la gloire » (p. 107).

5 L'auteur brosse également un tableau très vivant des intrigues auxquelles Guibert se trouve lié malgré lui en raison de ses liens avec de Broglie et avec Dumouriez, dont J.P. Bois le biographe récent de Dumouriez, nous avait déjà donné un aperçu.

6 En 1774, avec le nouveau règne, Guibert peut croire que son heure a sonné. En 1775, il se marie, mariage d'établissement comme il est de règle dans ce milieu, qui se transforme en union heureuse, l'épouse de Guibert, première thuriféraire de la pensée de son mari, devenant très rapidement une collaboratrice. En effet, Guibert se partage entre son régiment et l'écriture. Il rédige une nouvelle tragédie Les Gracques, jamais représentée, mais dans laquelle Ethel Groffier pense qu'il y a un écho de préoccupations révolutionnaires, fait l'Éloge du maréchal de Catinat pour le concours de l'Académie pour lequel il n'obtient que le premier accessit, ce qui est pour lui un échec. En même temps, la représentation de sa première tragédie à Versailles est également un échec malgré la faveur de la reine. Heureusement, il est appelé au Ministère de la Guerre auprès du nouveau ministre, le comte de Saint-Germain ; il pense pouvoir donner toute sa mesure et accomplir les réformes militaires dont il rêvait déjà dans son Essai général de tactique. Malheureusement, un concours de circonstances dévoya les intentions premières, ce que l'auteur analyse très finement. On (c'est-à-dire les adversaires de toute réforme) en rendit Guibert responsable. Il s'obstina à travailler à une réforme qui lui semblait de plus en plus problématique et, par deux mémoires militaires et politiques adressés au roi, il estime la guerre contre l'Angleterre inévitable et dit comment il faut s'y préparer.

7 C'est alors que Mademoiselle de Lespinasse, qui n'avait cessé depuis son mariage de l'accabler de reproches, meurt et Guibert avec magnanimité écrit l'Éloge d'Eliza, à la mémoire de son amie. Cette mort représente un tournant dans la vie de Guibert, contemporain de l'affaiblissement de la position de Saint-Germain et de l'annulation de ses réformes. Guibert redevient colonel dans le régiment de Neustrie. 
8 La deuxième partie, dénommée «Les années de réflexion" le voient revenir à une relative obscurité qui est aussi un temps d'approfondissement de sa pensée. Il écrit l'Éloge de Michel de l'Hospital qu'il ne présente pas au concours de l'Académie et qu'il publie anonymement.

9 Il voulait faire passer un message suffisamment clair et critique pour n'avoir aucune chance d'être accepté par l'Académie. Cet écrit rencontra un très grand succès, beaucoup plus que l'éloge officiel de l'abbé Rémy. Cela retarda l'élection de Guibert à l'Académie pour plusieurs années.

Désormais quand Guibert fréquente un salon, c'est un salon politique, notamment celui des Necker, ce qui accélère la maturation de sa pensée politique. Plus tard, il écrivit à la louange de la future Madame de Staël Zulmé, morceau traduit du grec. Le commencement des hostilités avec l'Angleterre lui donne l'occasion de parfaire et de nuancer sa pensée stratégique, notamment dans Défense d'un système de guerre moderne qui, bien plus qu'une tentative de trancher la controverse entre ordre mince et ordre profond, est un livre politique, en même temps qu'une autocritique lucide de l'Essai général de tactique. Allant sans cesse de la théorie à la pratique, il rédige de nouveau un plan général de guerre contre l'Angleterre, conservé à l'état de manuscrit. Mais la possibilité de servir son pays en Amérique lui échappe: le régiment de Neustrie devait faire partie des troupes de Rochambeau, mais resta à quai. Guibert, déçu, se fait historien dans L'histoire de la constitution militaire de la France. Toutefois, il était maintenant un personnage dont on ne cessait de parler même s'il était controversé et - consolation à ses déceptions mais aussi aliment à son besoin d'action et d'application de ses idées « sur le terrain »il seconde son père nommé gouverneur des Invalides et va inspecter en province les compagnies d'invalides dont les conditions de vie étaient déplorables. Enfin, il entre à l'Académie en 1786. L'année suivante, paraît un ouvrage inspiré par la mort de Frédéric II, L'Éloge de Frédéric, tout à la fois politique et militaire.

11 La troisième partie, "Le testament militaire ", est consacrée aux dernières années de Guibert. Officier le plus en vue de l'armée française, il ne parvient pas cependant, on dirait une fois de plus, à un rôle de premier plan. Certes, il devient rapporteur du Conseil de la Guerre créé en 1787 ; il prépare et rédige toutes les décisions du Conseil... mais ainsi il va être critiqué même pour celles avec lesquelles il n'est pas d'accord, d'autant qu'il reste silencieux quand l'ordonnance de 1788 confirme l'édit de 1781 sur l'exigence des quatre quartiers de noblesse. L'hostilité due à ces réformes se reporte sur Guibert et explique qu'il n'ait pas été élu aux états généraux quand il tenta d'être député de la noblesse du Berry. Il avait rédigé Discours à l'assemblée des trois ordres du Berry, dans lequel il se montre modérément réformateur; à la suite de cet échec, il ne fait donc pas partie du comité militaire de l'assemblée alors que celui-ci s'inspire des rapports de Guibert de même que le grand règlement du 1er août 1791 est issu des travaux du Conseil de la Guerre où on sait le rôle qu'il tint.

12 Il vivra les premiers temps de la Révolution en espérant encore un compromis entre les élites et entre le roi et celles-ci. Surtout, il rédige ce que nous-mêmes avons appelé son " chant du cygne », le traité intitulé De la force publique, auquel E. Groffier consacre avec raison une longue étude et dans laquelle elle voit avec raison également sa plus belle œuvre, car elle est l'aboutissement de la vie d'un homme qui n'a peut-être pas donné sa pleine mesure avant de s'éteindre encore jeune en mai 1790.

13 Madame de Guibert, dépositaire de la pensée de son mari et voulant lui rendre justice, a la malencontreuse idée de publier, entre autres, les Lettres de Mademoiselle de 
Lespinasse qui devaient faire tant de mal à la réputation de leur destinataire et occulter une œuvre et une pensée dont E. Groffier veut montrer la force et l'originalité. Le livre s'achève par une bibliographie intéressante où l'on s'étonne cependant de ne pas voir les ouvrages d'Isser Woloch à propos des Invalides et où l'on aimerait voir tous ceux qui, écrits en français, sont consacrés à l'histoire militaire de la Révolution et de l'Empire dont l'auteur reconnaît l'influence que joua Guibert sur elle. 